\title{
STUDY AND APPLICATION OF SOFTMAN COMMUNICATION MODEL
}

\author{
Zhonggui $\mathrm{Ma}^{1}$, Bin $\mathrm{Ye}^{1,2}$, Guangping Zeng ${ }^{3}$, Xuyan $\mathrm{Tu}^{3}$ \\ 'The Department of Automatic Control, Beijing Institute of Technology, Beijing $100081 ;{ }^{2}$ \\ Taiyuan University of Technology, Taiyuan 030024; ${ }^{3}$ School of Information Engineering, \\ University of Science and Technology Beijing, Beijing 100083
}

\begin{abstract}
By analyzing present communication model of multi-agent and SoftMan's characteristics, a SoftMan communication model which combined centralizeddecentralized mode with direct-indirect mode was presented base on multilevel hierarchical architecture of Large System Cybernetics. This model can assign task and deal with information logically. It can optimize scheduler, decentralize danger, and improve the speediness and security of communication of SoftMan system. Using this communication model can make system design more easily. A good application has been performed in digital gas field.
\end{abstract}

Key words: SoftMan Communication, DAI(Distributed Artificial Intelligence), Digital Gas Field, Multi-Agent System

\section{INTRODUCTION}

The term "SoftMan" was first put forward in the second bibliography. Its basic concept is developing a king of automated intelligent tool named "SoftMan" in the internet on the basis of Artificial Intelligence, Artificial Life and Distributed System, combined intelligent robot, intelligent net with Multi-Agent. This king of SoftMan can move freely, and deal with some tasks automatically using information push-pull technology. It can act as certain office clerk, such as safety policeman, garbage collector, information waiter in the internet, and so on.

In a SoftMan system, SoftMan can solve distributed problems by coordination and cooperation. However, communication is a bridge for 
coordination and cooperation. Communication in the SoftMan system includes communication between SoftMan and SoftMan platform, communication between SoftMan and SoftMan.

For this purpose, SoftMan communication language and communication protocols based on Speech Act theory must be built.

There are three main problems as follows need to be solved now:

1) Communication Management: After communication intention is produced, it usually can't be finished by one message; and there are logical relations between messages. The right understanding to a message relies on not only message contents and communication protocols, but also communication history. So it needs to manage communication process so as to combine communication content with cooperation.

2) Communication Language: Communication language is the media of message transferring and knowledge exchanging of SoftMan. All SoftMans share syntax, semantics and pragmatics of communication language.

3) Communication Protocols: The communication protocols refer to generalized communication protocols, which include high level communication protocols and low level transportation protocols. High level communication protocols describe basic processes of communication and all possible instances of corresponding messages, and they are usually described by finite state machine. Low level transportation protocols refer to transportation control in practical communication, such as TCP, SMTP and HTTP, and son on.

\section{COMMON COMMUNICATION MODEL}

Prof. Xuyan Tu has put forward three kinds of information structure of Multi-Agent System: Centralized Information Structure, Decentralized Information Structure and Hybrid Information Structure. Based on these structures, there are two common communication models: Client/Server Model(for short C / S Model) and Point-To-Point Model(for short P2P Model).

\subsection{Client/Server Model(C / S Model)}

Agent Communication is indirect in the communication system based on $\mathrm{C} / \mathrm{S}$. Model. There is a central server in this model, all agents only communicate with the central server and can't communicate one another, so agent communication relies on the central server to achieve communicating one another and sharing resource. This model usually use "blackboard" as communication mode. Figure 1 shows the block diagram of this model. 
The characteristics of $\mathrm{C} / \mathrm{S}$ Model as follows:

(1) Centralized Information

All agents only communicate with the central server and can't communicate one another. The central server controls the access rights of all agents, only registered agents can exchange information and share resource, which is in favor of allotting information resource reasonably.

(2) Simple Structure

The structure of this model is simple, so it is easy to achieve, diagnose and maintain.

(3) Centralized Load

Because information is centralized, load is centralized. As the number of agents increase, storage space of the central server and communication load between all agents and the central server also increase. The performance of server and network bandwidth will be the bottleneck of multi-agent system.

(4) Centralized Danger

Because information is centralized, danger is centralized. When the central server fails to run, it will lead to crash of multi-agent system. So reliability of $\mathrm{C} / \mathrm{S}$ Model is weak.

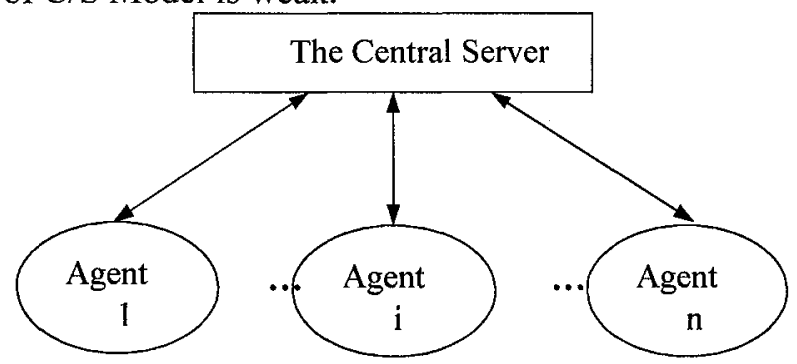

Figure 1. the block diagram of C/S Model

\section{$2.2 \quad$ Point-To-Point Model(P2P Model)}

Agent communication is direct in the communication system based on P2P Model, which need not transmit by the central server. This model uses decentralized communication structure and usually uses message as communication mode. Figure 2 shows the block diagram of this model.

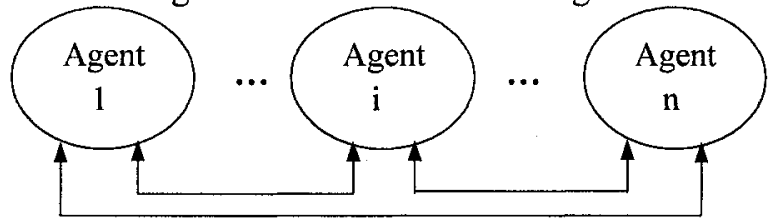

Communicate one another

Figure 2. the block diagram of $\mathrm{P} 2 \mathrm{P}$ Model 
The characteristics of $\mathrm{P} 2 \mathrm{P}$ Model as follows:

(1) Decentralized Information

There is not central server. All agents directly communicate one another with message. Any agent can be a sender or a receiver of message. This model seems like full interlinkage model of net model, so it is the same as peer to peer system. Because any two of agents have the direct access, they can communicate directly.

(2) Balanced Load

Multi-agent system doesn't depend on certain special node when it runs, so its load is balanced, and reliability of this model is strong.

(3) Harmonious Communication

Decentralized information structure needs to define communication protocols, formats of message, transmitted mechanism and communication language (including syntax and semantics).In this way, exchanging information and sharing resource can be easily done among agents.

(4) Difficult to Achieve

First, because each agent saves status information of all agents, it increases local storage load. Second, any inner status change of an agent must inform other agents, it increases communication load. Third, every agent must deal with correlative calculation of controlling and scheduler, so it increases calculating load of multi-agent system. Because all of these need perfect low level communication protocols, it is difficult to achieve.

\section{SOFTMAN COMMUNICATION MODEL}

From the two models discussed above, we can learn that the communication system can't satisfy the multitasking requirement of SoftMan only based on C/S Model or P2P Model. Therefore, this paper puts forward SoftMan communication model with multi-level hierarchical architecture on the basis of C/S Model and P2P Model. This model has the merits of the two models discussed above. Figure 3 shows the block diagram of this model.

The goal of SoftMan Design is to simulate behavior of human society, extend and prolong human intelligence, so SoftMan should be marked off according to role and ability of human society. Different SoftMan has different ability and plays a different role. We mark off one group with commonness, and each group assigns a group leader who manages other SoftMan of his group and communicates with other groups by the central server. In the SoftMan group of figure 3, we supposed that there are $n$ groups of SoftMan, such as Group 1, Group 2, ......, Group n, the SoftMan number of each group is $r, s, \cdots \cdots, t$ accordingly, and the group leader of each group 
is $\mathrm{GL}_{1}, \mathrm{GL}_{2}, \cdots \cdots, \mathrm{GL}_{\mathrm{n}}$ accordingly. Communication between group and group uses $\mathrm{C} / \mathrm{S}$ Model, respectively group leader of each group is delegated to communicate with group leader of other groups, and the central server save name, address, ability and status of group leader of all groups, etc. Communication within one group uses P2P Model which harmoniously communicates with full interlinkage mode.

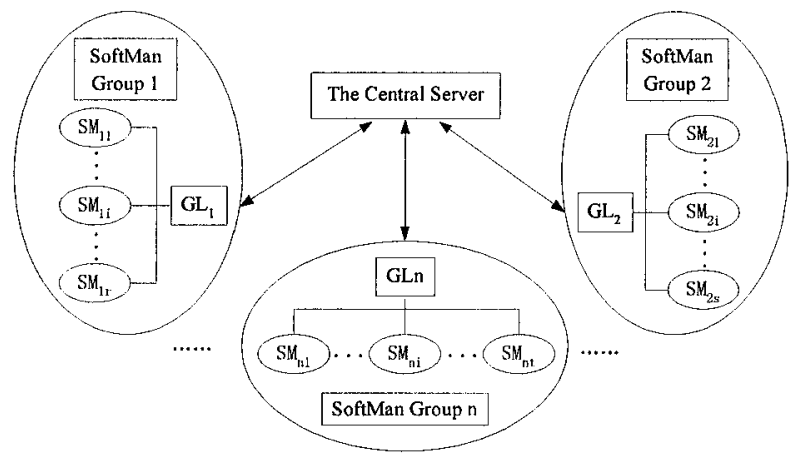

Figure 3. SoftMan Communication Model

Because SoftMan communication model combined centralizeddecentralized mode with direct-indirect mode, it can learn from others's strong points to offset one's weakness, assign task and deal with information logically. It can also optimize scheduler, decentralize danger, and improve the speediness and security of communication of SoftMan system. Using this communication model can make system design more easily.

\section{COMMUNICATION LANGUAGE AND COMMUNICATION PROTOCOLS OF SOFTMAN}

In this paper, KQML(Knowledge Query and Manipulation Language) is used as communication language and is expanded in security. KQML is a kind of high-level and message-oriented communication language and communication protocols for agent, it supports knowledge-sharing of runtime and coroutine problem. KQML messages do not merely provide communication sentences, they are sent to recipients with an attitude about the content(assertives, directives, commisives, declaratives, and expressives). An attitude of content is conceptualized to primitives that are called performatives. It provides an extensible set of performatives, using it we can build high-level agent communication model, such as Contracts Nets. It uses facilitator to communicate with different agents, and facilitator is also a king of special agent. 
KQML is a kind of hiberarchy language. Conceptually, KQML message can be viewed as being divided into three layers: the content layer, the message layer, and the communication layer. The KQML structure is illustrated in Figure 4. The content layer bears the real content of the message in the program's own representation language. KQML may use any form of languages(such as ASCII character strings, binary code), so its realization form is independent of meanings of the content layer. The message layer determines the kinds of interaction between KQML-speaking agents. The main function of the message layer is to identify the protocol to be used to deliver the message and to supply a speech act or performative that the sender attaches to the content. The speech act indicates whether the message is an assertion, a query, a command, or any other of a set of known performatives. The communication layer encodes a set of features to the message that describe the lower-level communication parameters, such as the identity of the sender and recipient, and a unique identifier associated with the communication.

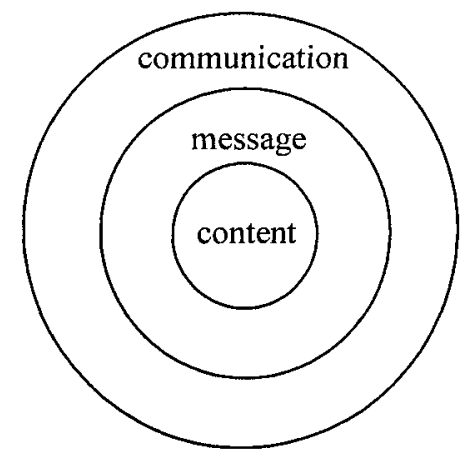

Figure 4. KQML structure

\section{SOFTMAN APPLICATION IN DIGITAL GAS FIELD}

The conformity of multi-source complex information is the core in the key technology of digital gas field, it directly restrict the success of production control, decision-making and data mining, and its purpose is to achieve the conformity of multi source and multilayer data, and make existing application exchange data one another. Because of lacking of standard data format, point-to point way is inefficient when every application get data from other applications. For example, as data formats of certain application have been changed, other applications using this data must change accordingly. One way to solve this problem is to build uniform 
data format in data exchanging center, and build corresponding relation between uniform data format and its own data format in every application accordingly.

Therefore, a kind of standard data format should be built. At the same time, there are many existing application software and many kings of database, such as text file, FoxBASE, Access, SQL Server, Sybase, Oracle, and so on, so many application interfaces and data interfaces should also be built. It is difficult to carry into execution. Thus we present the data exchange method using SoftMan technologies. The data exchanging structure is illustrated in Figure 5. Data exchanging center communicates and exchanges data (including uploading data and downloading data) by the interface SoftMan of data exchanging. First, the interface SoftMan of data exchanging decides to call certain SoftMan group by parsing message header. Second, the group leader of SoftMan group receiving message shares message by broadcasting. Third, query SoftMan queries data from corresponding database according to query condition and saves the data in the data cache. Fourth, validating SoftMan checks the validity and rationality of data and saves the result. Fifth, converting SoftMan generates standard data according to corresponding relation between uniform data format and data format of current application. Sixth, executing SoftMan packages the data and informs the group leader to transfer data. The data of different locations and different formats can access one another by the data exchanging center, and the data exchanging center integrates correlative application systems and forms the basic platform of digital gas field.

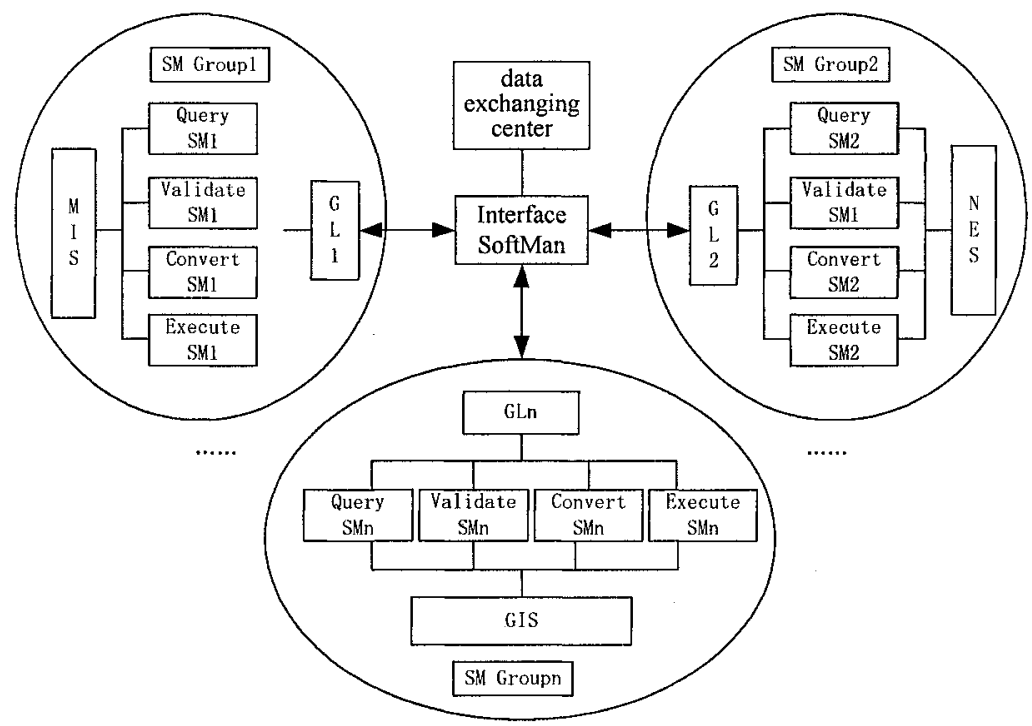

Figure 5. data exchanging structure 
This structure belongs to LCN like stelliform net, and it is easy to expand in structure. It can build multilevel data exchanging center so as to support fargoing Wide Area Information Server. Using SoftMan technologies, greatly predigest design difficulty and it is easy to maintain. A good application has been performed in digital gas field.

\section{CONCLUSION}

With the development of computer and network technology, internet has been anywhere. However, more and more people need to provide safe, shortcut and humanity information services in tremendous amount ofvast net world. Many experts and scholars have done many predominant work, on the basis of these work, a SoftMan communication model which combined C/S Model with P2P Model was presented base on multi-level hierarchical architecture of Large System Cybernetics. This model can assign task and deal with information logically. It can optimize scheduler, decentralize danger, and improve the speediness and security of communication of SoftMan system. Using this communication model can solve communication problems of distributed system effectively.

\section{NOTES}

Foundation item: National Science Foundation of China (No.60375038)

\section{REFERENCES}

1. Xuyan Tu, Large System Cybernetics, Defense Industrial Publishing House, 1994, Beijing.

2. Guangping Zeng and Xuyan Tu, "SoftMan", < Progress of Artificial Intelligence in China>, Proceedings of 10th CAAI Conference, Beijing University of Posts and Telecommunications Publishing House, 2003, Beijing.

3. Zhongzhi Shi , Intelligent Agent and its Applications, Science Publishing House, 2000, Beijing.

4. Xuyan Tu and Junping Du etc., Multi-Agent System, Software World, Aug. 2000, Beijing.

5. Xinyu Feng and Xianping Tao etc., An improved mobile Agent communication algorithm, Chinese Journal of Computers, 2002, 25(4):357 364, Beijing.

6. Thome, M. Multi-tier communication abstractions for distributed multi-agent systems. Integration of Knowledge Intensive Multi-Agent Systems, 2003. International Conference on, 30 Sept.-4 Oct. 2003 Pages:209 - 214 